\title{
Transatlantica
}

Revue d'études américaines. American Studies Journal

\section{De l'utopie socialiste au réalisme chrétien}

Reinhold Niebuhr et le New Deal

\section{Isabelle Richet}

\section{(2) OpenEdition}

Journals

Édition électronique

URL : http://journals.openedition.org/transatlantica/178

DOI : $10.4000 /$ transatlantica. 178

ISSN : $1765-2766$

Éditeur

AFEA

\section{Référence électronique}

Isabelle Richet, « De l'utopie socialiste au réalisme chrétien », Transatlantica [En ligne], 1 | 2006, mis en ligne le 24 mars 2006, consulté le 29 avril 2021. URL : http://journals.openedition.org/transatlantica/ 178 ; DOI : https://doi.org/10.4000/transatlantica.178

Ce document a été généré automatiquement le 29 avril 2021.

\section{(c) (i)}

Transatlantica - Revue d'études américaines est mis à disposition selon les termes de la licence Creative Commons Attribution - Pas d'Utilisation Commerciale - Pas de Modification 4.0 International. 


\title{
De l'utopie socialiste au réalisme chrétien
}

\author{
Reinhold Niebuhr et le New Deal
}

Isabelle Richet

1 En m'intéressant à l'attitude face au New Deal de Reinhold Niebuhr, théologien vedette du protestantisme libéral et une des figures marquantes de la gauche intellectuelle new-yorkaise des années trente, je voudrais tenter d'expliquer le constat amer que dressait John C. Bennett, l'un de ses amis en théologie et politique, en 1939:

"One of the hardest facts to face is that the success of the most promising political forces in American life must be in spite of the opposition of the majority of the members of the Protestant churches » (cité par Miller 1958, 75)

Alors que tout au long du XIX ${ }^{\mathrm{è}}$ siècle le protestantisme avait embrassé, et souvent initié, une multitude de mouvements de réforme; alors que nombre de ses théologiens et de ses pasteurs s'étaient engagés dans le mouvement progressiste du début du siècle, dans les années trente, une majorité des responsables protestants et de leurs ouailles adoptèrent une position conservatrice et parfois une hostilité ouverte face à la plus importante expérience de transformation sociale de l'histoire des Etats-Unis (Cawardine 1993 ; McLoughlin 1978). Les chiffres sont sans équivoque : en 1932 et 1936 pratiquement tous les hebdomadaires protestants soutiennent les candidats républicains ; en 1932, 1936 et 1940 la majorité des pasteurs se prononcent contre FDR et ses réformes; en 1932 une majorité des protestants votent pour Hoover et même dans l'élection triomphale de 1936 ils n'apportent à FDR qu'une très étroite majorité alors que les électeurs catholiques et juifs le choisissent à plus de $80 \%$; malgré le soutien apporté par le Federal Council of Churches, structure bureaucratique qui ne parle que pour elle-même, la majorité des dénominations se montrent fort circonspectes quand elles ne dénoncent pas le collectivisme et l'athéisme triomphants à Washington, comme le fait la Southern Baptist Convention, principale dénomination du Sud démocrate (Gustafson 1988, 285-297 ; Miller 1958, 75, 117-123 ; Menendez 1972, 60-75 214-215).

Plusieurs hypothèses peuvent être avancées pour expliquer cette attitude : l'adhésion des classes moyennes protestantes à l'idéologie du rugged individualism et du 
laissez-faire incarnés par le parti républicain - menant même chez les plus riches à une haine profonde de F.D. Roosevelt-; le sentiment d'être socialement et politiquement déplacées par une nouvelle majorité urbaine et ethniquement mixte courtisée et rassemblée par le président démocrate; un ressentiment ethno-culturel incommensurable à l'égard de celui qui s'était allié au candidat catholique d'origine irlandaise Al Smith lors de la campagne de 1928, puis du président qui abolit la prohibition dès son élection (Reichley 1985, 209-210 ; Baltzell 1964, 227-248; Muelder 1961, 173). Mais il faut aussi y ajouter l'échec des responsables de ce que l'on a appelé l'Evangile social, ces pasteurs et théologiens libéraux qui, depuis le début du siècle avaient essayé de tisser de nouveaux rapports entre religion et politique dans le contexte de la société industrielle. C'est ce dernier point qui m'intéresse ici, car c'est en opposition à leur idéalisme naïf que Reinhold Niebuhr engagea une réflexion qui le mena, selon ses propres termes "à une révolte graduelle contre toute la culture libérale de l'époque ", refuge confortable de la bourgeoisie triomphante (Niebuhr 1939a). Il s'agissait au départ d'une réflexion socio-politique qui le porta à utiliser les outils marxistes d'analyse de la société en termes de classes, de rapport de forces et de pouvoir, et à militer au Parti socialiste de Norman Thomas pour construire une alternative au libéralisme du New Deal. Mais elle s'accompagna d'une réflexion théologique, qui réintroduisit le poids du péché originel comme limite absolue de toute l'expérience humaine et déboucha sur le rejet de toutes les utopies terrestres et, bientôt, sur la révision de son radicalisme de la première moitié des années trente et l'acceptation du New Deal comme la meilleure approximation de la justice sociale dans un monde imparfait où on ne pouvait espérer voir se réaliser ni les lendemains qui chantent des socialistes et des communistes, ni le Royaume de Dieu annoncé depuis le début du siècle par les prophètes de l'Evangile social.

Reinhold Niebuhr a souvent été appelé le théologien du paradoxe et son attitude dans les années trente justifie sans aucun doute une telle qualification, alors que par réalisme politique il s'opposa d'abord au New Deal mais, par réalisme théologique, il finit par s'y rallier.

5 Pour saisir cette évolution je présenterai d'abord sa prise de conscience critique de l'idéalisme du Social Gospel pour étudier ensuite son tournant radical du début des années trente puis son tournant orthodoxe de la fin de la décennie. Une telle approche, adoptée par souci de clarté, risque d'appauvrir la dialectique serrée entre analyse sociale et théologique qui sous-tend sa réflexion dès le départ, risque que je chercherai à minimiser en soulignant comment ces deux filons de la pensée de Reinhold Niebuhr se nourrissent mutuellement et déterminent ses choix politiques au cours de la décennie.

6 Sa critique politico-sociale du Social Gospel est une critique de l'intérieur. C'est au sein des milieux théologiques libéraux que Reinhold Niebuhr a fait ses premières armes intellectuelles. Après des études à l'université de sa petite dénomination évangélique allemande dans l'Illinois (German Evangelical Synod of North America), il poursuivit sa formation au séminaire de Yale en 1913 puis, en 1915 fut nommé pasteur à Détroit, avant d'obtenir, en 1928, un poste de professeur d'éthique au prestigieux Union Theological Seminary de New York, bastion du libéralisme religieux et politique, où il restera jusqu'à la fin de sa carrière, intégrant dès son arrivée les cercles de la gauche socialiste de la ville (Fox 1985, 14-28). 
7 Formé au sein des milieux protestants libéraux, Reinhold Niebuhr en partageait au départ bien des analyses et des principes. Comme les prophètes du Social Gospel, qui avaient pourtant introduit la notion de péché social s'incarnant dans des institutions économiques et politiques injustes, il pensait, en fin de compte, que seule la conversion des individus à l'amour chrétien apporterait une solution aux conflits de classe, et permettrait de créer une société harmonieuse à l'image du Royaume de Dieu (Fox 1985, 23 ; Meyer 1988, 34-35).

8 Pourtant il commença assez vite à réévaluer cette approche à la lumière de la Première Guerre mondiale, de son expérience des conflits sociaux à Détroit, puis de la crise de 1929 et des nuages qui s'amoncelaient en Europe, selon une démarche très pragmatique d'élimination graduelle des analyses et actions jugées inefficaces, qui explique le côté parfois contradictoire et toujours fortement polémique de ses prises de position.

9 La Première Guerre mondiale puis l'échec de la croisade wilsonienne avaient mis à mal la croyance aveugle dans le progrès et dans la perfectibilité de l'homme, qui avait nourri les idéaux progressistes et les espoirs de l'Evangile social. Reinhold Niebuhr, pour sa part, en retira des leçons pessimistes sur les rapports entre nations et groupes sociaux, renforcées par un voyage en Allemagne après l'occupation de la Ruhr, leçons qui le porteront de plus en plus à se poser la question du pouvoir en termes politiques et non plus éthiques. La guerre, et surtout l'adhésion outrancière des Eglises américaines à l'hystérie anti-allemande et au militarisme (Abrams 1933), le convainquirent aussi de remettre fondamentalement en cause l'identification entre religion et nation établie par le protestantisme depuis les premières décennies de la république. Par sa critique du nationalisme, religion civile qui déplace la transcendance vers la nation, donnant ainsi « une signification éternelle à des fins qui n'ont pas de valeurs éternelles ", il appelait à opérer une distinction entre la sphère du religieux, qui s'intéresse aux questions ultimes, et la sphère du politique qui gère les relations sociales entre les hommes, premier pas vers le rejet de la nouvelle synthèse entre religion et culture que les prophètes du Social Gospel s'étaient efforcés de rétablir depuis le début du siècle (Niebuhr 1928a).

10 Pasteur d'une congrégation de la capitale industrielle des Etats-Unis, il prit graduellement et directement conscience de la nature des conflits industriels et de leurs causes qui rendaient bien irréelle l'image de la communauté idéale propagée par le Social Gospel, dans laquelle l'amour chrétien devait transcender les intérêts de classe. Lui-même avait, dans un premier temps, adhéré à cette vision et défendu pour l'Eglise un rôle d'arbitre neutre entre Capital et Travail. S'il envisageait un degré de démocratie industrielle, il affirmait aussi, dans la tradition du Social Gospel, que « pour entraîner une renonciation volontaire à sa richesse de la part de la classe capitaliste " l'Eglise devait «se démarquer de la haine de classe du prolétariat » et de "son mépris cynique du pouvoir de l'altruisme dans la nature humaine" (cité par Fox 1985, 71). Pourtant, face aux grèves de l'après-guerre, alors que le Federal Council of Churches, principale institution du Social Gospel, réaffirmait, dans son rapport The Church and Social Reconstruction (1919), sa neutralité face aux forces sociales en présence et appelait les capitalistes à sacrifier leurs privilèges et les ouvriers à renoncer à leur conscience de classe et aux grèves, Reinhold Niebuhr, lui, commençait à contester cette approche équilibrée qui ne tenait pas compte des inégalités de pouvoir entre patrons et ouvriers. C'est alors qu'il commença à développer son analyse de "groupe »-sans utiliser 
encore le terme de classe - qui le mènera bientôt à emprunter les outils d'analyse marxiste. Il remarquait en effet que :

All human groups are essentially predatory, and tend to hold desperately to their privileges against the pressure of the underprivileged who demand a fancier share of the blessings (Niebuhr 1928b).

11 Si l'on souhaitait mettre fin aux conflits sociaux, il fallait en fait transformer toute la structure de pouvoir du système industriel. De même, aux chrétiens qui rejetaient les grèves comme des formes d'action anti-sociales, il rappelait que «l'organisation de l'industrie moderne que les grèves perturbent et remettent en cause est aussi anti-sociale que les grèves elles-mêmes ", et il les invitait à prendre clairement parti pour le mouvement ouvrier, dénonçant comme illusoires les appels moraux aux capitalistes pour qu'ils acceptent volontairement de se départir de leur richesse (Federal Council of Churches 1919 ; Niebuhr 1922).

De même, la vie politique locale lui fit prendre conscience des réalignements politiques en cours, liés à la nature de plus en plus pluraliste de la société américaine, alors que, avec la Prohibition et les campagnes du Ku Klux Klan des années vingt, les forces du protestantisme tentaient, parfois de façon musclée, de maintenir une société culturellement homogène modelée par les valeurs WASP. Il avait été choqué, lors des élections municipales de Détroit en 1925, de constater que ses coreligionnaires avaient décidé de soutenir le candidat du Klan contre un catholique « mouillé » et il avait, pour sa part, appelé ses ouailles à " porter un coup décisif à l'organisation encagoulée » (Fox 1985, 91). Au cours des années suivantes il accentua ses critiques de la Prohibition - qu'il avait d'abord acceptée -, reprochant à «la majorité nordique arrogante » de ne pas saisir les changements culturels en cours, et aux Eglises d'avoir recours au bras séculier de l'Etat pour imposer à tous une position morale qu'elles n'arrivaient pas à convaincre les gens d'adopter par la persuasion (Niebuhr 1928c). Une critique qui l'amena finalement, en 1928, à trouver le catholique mouillé Al Smith, contre lequel toutes les Eglises protestantes s'étaient liguées, préférable à Hoover, car il lui semblait plus important de placer la question de la justice sociale, mieux représentée par le démocrate Smith, avant celle de la pureté personnelle. Mais lui-même appela déjà à cette occasion à voter pour le socialiste Norman Thomas.

13 Ainsi, quand il arriva à New York à la veille de la dépression, Reinhold Niebuhr avait déjà opéré plusieurs ruptures fondamentales avec la démarche du Social Gospel, sans en avoir encore nécessairement tiré toutes les implications politiques et théoriques. Tout comme les prophètes du Social Gospel il se souciait avant tout d'établir un rapport satisfaisant entre religion et politique, mais avait conclu que leur approche était doublement inefficace. Religieusement d'abord car, en tentant de légitimer la religion par son utilité sociale, elle émoussait les vérités ultimes intemporelles dont celle-ci était porteuse. Politiquement ensuite, car en se donnant pour but la vision idéalisée du Royaume de Dieu et en ne proposant que des moyens d'action en accord avec cet idéal, elle interdisait d'intervenir de façon crédible dans une société dominée par des intérêts de classe irréductibles, comme en témoignait son échec à convertir les patrons comme les ouvriers.

14 C'est dès lors dans le feu de l'action politique qu'il approfondit sa rupture et accentua son tournant politique radical, tout en poursuivant sa réflexion théologique, restant fidèle à sa démarche pragmatique qui le poussa d'abord à définir les moyens d'action efficaces pour, ensuite, dessiner petit à petit les objectifs réalistes de cette action. 
15 New York, avec son cosmopolitisme culturel et son éventail de groupes politiques plus ou moins radicaux, lui offrait un théâtre d'action qui lui permettait de concrétiser plus systématiquement ses engagements intellectuels. En même temps, son intégration comme professeur à Union Theological Seminary lui offrait un solide ancrage religieux qui lui permettait de combiner militantisme et réflexion théologique.

Il se lança dès son arrivée à New York dans un activisme qui illustrait autant, sinon plus, sa volonté d'agir politiquement que la cohérence de ses choix et la clarté de ses objectifs. En tant que professeur, il adhéra au syndicat des enseignants de la ville, aux tendances de gauche affichées. Il adhéra également à la Fellowship of Reconciliation, organisation pacifiste d'inspiration chrétienne, au sein de laquelle il retrouva des amis protestants, comme Kirby Page et Sherwood Eddy, mais aussi des laïcs comme l'ancien pasteur A.J. Mustee qui avait quitté le clergé au début des années vingt pour donner la priorité à l'action politique. Il participa également à la League of Industrial Democracy, formation créée par le socialiste Norman Thomas, et à la League for Independent Political Action, aux côtés de libéraux plus ou moins à gauche, tels que John Dewey, Norman Thomas, W.E.B. DuBois, Villard - le rédacteur en chef de The Nation - ou A. J. Mustee, qui tous soutenaient la formation d'un troisième parti, sans pour autant encore s'accorder sur sa configuration politique. Enfin, en septembre 1929, il adhéra au Parti socialiste de Norman Thomas, dont il devint un militant actif, se présentant même sur une liste de ce parti aux élections pour le Sénat de l'Etat de New York en 1930 (Fox 1985, 116-117 ; Meyer 1988, 128 ; Pells 1974, 52-54).

C'est avant tout son souci d'efficacité qui l'amena a établir fermement la primauté du politique sur l'éthique dans l'action sociale. Dans son ouvrage Does Civilization Need Religion?, il abandonnait l'idée, propre au Social Gospel, que l'Eglise en tant que telle pouvait mener le mouvement de rédemption de la société. Si la religion pouvait nourrir la critique sociale à partir d'une vision idéale, c'est aux laïcs qu'il revenait d'agir en politique en fonction des rapports de force concrets et en adoptant les moyens dictés par la réalité et non par l'idéal (Niebuhr 1927, 223). En politique, en effet, les hommes agissaient en fonction d'un futur pragmatique à l'opposé du Social Gospel qui opérait à partir d'un futur idéal. Logiquement, son engagement dans la League for Independent Political Action concrétisait son choix de la primauté du politique et d'une action pour laquelle les individus se regroupaient indépendamment de leurs origines culturelles, à partir de leur compréhension des enjeux de pouvoir, alors que le Social Gospel voyait l'Eglise non seulement comme seul outil d'intervention politique pour les chrétiens, mais également comme la préfiguration de la communauté idéale qui devait, à terme, absorber en son sein le Capital et le Travail (Meyer 1988, 128).

Son choix était également dicté par la conscience de la réalité sociologique du protestantisme, qui était depuis le départ le talon d'Achille du Social Gospel. Dans le Nord $76 \%$ et dans le Sud $56 \%$ des protestants appartenaient aux classes moyennes et supérieures. Du fait de sa conviction désormais bien établie que les groupes sociaux agissaient en fonction de leurs intérêts de classe, cette réalité sociologique - cette «captivité de classe »-du protestantisme l'amena à conclure qu'il existait une contradiction fondamentale entre les idéaux religieux d'harmonie sociale prônés par le Social Gospel et les intérêts économiques des membres de l'Eglise. Contradiction qui amena, en effet, la majorité des congrégations à s'opposer à Roosevelt et empêcha les pasteurs de s'identifier au New Deal, d'autant plus qu'ils étaient soumis à la pression organisée des conservateurs au sein des dénominations dont les bureaucraties 
nationales auraient pu être tentées de soutenir les efforts du président démocrate (Christian Century 1935).

19 Sa décision de s'engager dès le début de la crise et jusqu'à la fin de la décennie dans le parti socialiste était également dictée par le rejet de ce qu'il considérait comme l'idéalisme apolitique impénitent de beaucoup de ses amis protestants libéraux de gauche au cours des premières années de la Dépression. Face à l'effondrement du système, le Christian Century, principal organe du Social Gospel dans les années Vingt et Trente, appela, sous la plume de son rédacteur en chef Charles Clayton Morrison, à un "réveil moral des capitalistes", qui devait mener à "une liquidation volontaire du système fondé sur la concurrence» (Christian Century 1931). Tout en prônant la planification de l'économie, Morrison se gardait bien de demander qui devait planifier et en fonction des intérêts de quel groupe social (Morrison 1932b). L'inconsistance politique du Century fut également illustrée par les contorsions de Morrison à la veille des élections de 1932, alors que d'un côté il rejetait les deux principaux partis au nom $\mathrm{du}$ "parti des désintéressés »-ce qui signifiait la communauté protestante qu'il considérait encore comme l'expression de la communauté nationale - , mais appelait finalement à voter pour le républicain Hoover, au nom de sa défense de la Prohibition qui faisait partie de la doctrine de l'Eglise (Morrison 1932a). En fait pour Morrison et ses amis, la politique n'avait aucune fonction distincte, et la christianisation de la société restait la solution à tous ses maux, alors que Reinhold Niebuhr était conscient que la crise que traversaient les Etats-Unis exigeait en permanence des décisions politiques concrètes.

C'est ce souci de réalisme qui l'amena à s'opposer également à deux groupes politiques protestants plus radicaux, animés par certains de ses proches amis. Le premier était la Methodist Federation of Social Service, dirigée par Harry Ward qui avait opéré un tournant socialiste et même communiste au début des années Vingt, avait passé une année en URSS en 1931-32 et en était revenu un ami convaincu de l'Union soviétique. Niebuhr partageait son analyse de la crise dans laquelle Ward voyait la faillite du système capitaliste - une analyse que Niebuhr lui aussi développa dans Reflections on the End of an Era (1934) - , son livre le plus anticapitaliste qui saluait l'effondrement finale de la bourgeoisie occidentale. Il partageait aussi sa critique du New Deal, dans lequel Ward ne voyait qu'un programme visant à sauver le capitalisme grâce à l'intervention de l'Etat. Mais il rejetait son idéalisme quasiment mystique qui le poussait à défendre la pureté de son but communiste et à rejeter tous les choix et compromis politiques tactiques nécessaires pour l'atteindre.

21 L'autre organisation était la Fellowship of Reconciliation-dont Niebuhr était membre - animée par son ami Kirby Page. Formée au début des années Vingt, elle avait pour objectif «l'abolition de la guerre » et prônait un pacifisme radical « où que cela menât et quoi que cela coûtât " (Page 1925,92). Ce pacifisme s'appliquait également aux conflits sociaux. Page appelait à un "pacifisme de classe ", une position qui, dans le contexte des tensions sociales des années trente, provoqua un débat au sein de la F.O.R. et à une scission en 1933, la majorité se prononçant contre le recours à la violence défensive par les ouvriers. Reinhold Niebuhr rompit alors avec cette organisation - ce fut aussi le cas d'A.J. Mustee qui rejoignit temporairement les trostkystes du Workers' Party - appliquant sa démarche réaliste au choix des moyens d'action. Dans Moral Man and Immoral Society (1932) il avait déjà laissé entendre que le choix des formes de lutte devait être pragmatique, c'est-à-dire politique, et non 
éthique. En conséquence, si l'on admettait que l'idée de la lutte pour la justice sociale était moralement justifiée, on ne pouvait pas tracer une ligne de démarcation nette entre la violence et la non-violence, et la violence ne pouvait pas être rejetée a priori (Niebuhr 1932, 172, 179). C'était la première formulation de son «réalisme chrétien » selon lequel un être moral pouvait être amené à utiliser des moyens qu'il jugeait immoraux si la réalité l'imposait, position qu'il réaffirma clairement dans l'article qui annonçait sa rupture avec la F.O.R. :

Modern capitalism breeds injustice by the disproportions of economic power which it tolerates and upon which it is based. We expect no basic economic justice without the destruction of the present disproportion of power and we do not expect the latter without a social struggle. Once we have accepted the reality of the social struggle, we do not think that we can stop where the middle portion of the Fellowship has stopped. We are unable to stop there because we can find no stable absolute in the shifting situation of the social struggle where everything must be finally decided in pragmatic terms. Therefore violence cannot be categorically excluded as a means (Niebuhr 1934a).

Voyant dans le communisme mystique de Ward d'un côté et le pacifisme radical de la F.O.R. de l'autre des attitudes qui, tout comme celle du Social Gospel, préféraient la défense de la pureté de l'idéal à la pertinence politique, il les rejetait également au nom du réalisme social, à un moment où la profondeur de la crise exigeait que les idéaux soient testés concrètement dans l'action.

Ce réalisme quant aux moyens s'appliquait également aux fins. Si, jusqu'ici, il s'était contenté de critiquer la vision proposée par le Social Gospel d'une société harmonieuse cimentée par l'amour chrétien, il lui fallait désormais lui opposer une alternative crédible. C'est ce qu'il fit dans son livre Moral Man and Immoral Society qui est sans doute son livre le plus laïque et même le plus marxiste. Comme son titre l'indique, il y opérait une nette distinction entre l'individu et la société, entre la moralité religieuse et la moralité politique, faisant exploser tout le sous-bassement théologique de l'approche politique du Social Gospel. Si les rapports entre les individus pouvaient être régis par l'amour et l'altruisme, quand on déplaçait l'attention vers les groupes sociaux, force était de constater que les relations étaient déterminées par les intérêts et les rapports de pouvoir. Dans une société divisée en classes aux intérêts antagoniques, la moralité religieuse n'était pas à même de contrôler les motivations égoïstes des groupes. Quand elle y réussissait - et il prenait l'exemple de Tolstoï - cela menait à des actions totalement inefficaces du point de vue politique (Niebuhr 1932, 262-270). Il en concluait que :

The social viewpoint stands in sharp contrast to religious morality when it views the behavior of collective rather than individual man, and when it deals with the necessities of political life. Political morality, in other words, is in the most uncompromising antithesis to religious morality.

En conséquence, l'objectif de la lutte politique ne pouvait pas être la réalisation des idéaux religieux, mais la justice. Et justice ne signifiait pas amour, mais égalité sociale (Niebuhr 1932, 259, 234).

Si la justice et l'égalité étaient les seuls objectifs crédibles de l'action politique, et de plus les seuls permettant de répondre à la crise présente, l'agent social susceptible de les incarner était la classe ouvrière, car ces objectifs correspondaient naturellement à ses intérêts de groupe. Et dans ses écrits de 1933 et 1934, Niebuhr parle souvent de « l'inévitabilité de l'avènement du pouvoir du prolétariat » (Niebuhr 1934c, chap. 11). 
Pourtant, rien ne laissait supposer que, au début des années Trente, la classe ouvrière américaine était disposée à incarner ces idéaux - et Niebuhr lui-même n'avait de cesse de critiquer le conservatisme de l'American Federation of Labor. Sa tactique politique rejoignait là sa réflexion théorique. Son problème, comme celui de beaucoup de ses amis, était que s'il existait bien une classe ouvrière aux Etats-Unis, il n'y avait pas de mouvement ouvrier digne de ce nom. Pour utiliser la terminologie marxiste, il y avait bien une classe en soi, mais pas une classe pour soi. La construction d'une force politique susceptible de remédier à cette carence était l'objectif de son engagement au sein du parti socialiste. En effet, le troisième parti que Niebuhr appelait de ses vœux ressemblait au Labour Party britannique, parti ouvrier de masse qui alliait un socialisme non doctrinaire à des valeurs culturelles issues de la religion - et d'ailleurs son choix du parti socialiste de Norman Thomas, ancien pasteur presbytérien qui avait opté pour la politique au début des années Vingt, avait aussi été motivé par le fait que celui-ci n'exprimait pas d'opposition de principe à la religion, contrairement à beaucoup d'autres militants de gauche de l'époque. Le parti socialiste devait ainsi être le noyau du futur parti de masse et sa tâche principale était d'éduquer la classe ouvrière à l'idée de la propriété sociale des moyens de production, condition d'un système garantissant l'égalité et la justice sociale (Niebuhr 1934c, ix). De ce point de vue, la tâche la plus urgente était de démonter les illusions nourries par le New Deal. Même si le capitalisme d'Etat valait mieux que l'anarchie du laissez-faire, Reinhold Niebuhr voulait ouvrir les yeux à ceux qui voyaient dans la National Recovery Administration une forme de contrôle social, alors qu'il s'agissait d'utiliser l'Etat, qui n'était pas neutre mais contrôlé par les groupes dominants, pour remettre l'économie sur ses rails sans s'attaquer à la structure même du système capitaliste (World Tomorrow, 1933). Cette critique s'adressait à beaucoup de ses amis protestants libéraux qui, à l'instar du Federal Council of Churches et du Christian Century, soutenaient désormais le New Deal, n'hésitant pas, pour certains, à l'auréoler de leur vision idéaliste.

Les années 1933-35 représentent sans aucun doute la période la plus radicale de Niebuhr, celle où il utilisa les outils analytiques du marxisme, qui lui semblaient les mieux à même de faire sens de la crise et des insuffisances du New Deal (Niebuhr 1934, 135), celle où, mû par sa crainte de l'inertie sociale et son désir de construire une société juste, il donna la priorité à l'engagement politique aux côté des socialistes. Pourtant, le choix du parti réformiste, gradualiste, de Norman Thomas - qui lui avait d'ailleurs reproché son recours au marxisme dans Moral Man - signalait pour le moins certaines limites à ce radicalisme. Mais surtout, dès 1935-36, tout en continuant à soutenir le parti socialiste et à prôner une stratégie anticapitaliste, il tourna de plus en plus son attention vers une réflexion théologique qui, toujours au nom du réalisme, l'amena à abandonner petit à petit toutes ses prises de position de la première moitié de la décennie.

Bien qu'il n'ait jamais abandonné ses préoccupations religieuses - elles étaient mêmes, dans une certaine mesure, à la base de sa critique du Social Gospel-il expliqua lui-même, qu'il avait en fait procédé très graduellement, sous la pression des évènements internationaux, à une élaboration théologique à partir de ce qui n'était, au départ, qu'une critique sociale et éthique (Niebuhr 1939a). Cette évolution fut stimulée par une combinaison de facteurs idéologiques, pratiques et religieux. Si Moral Man était une critique de l'idéalisme du Social Gospel, son analyse du pouvoir des intérêts égoïstes des groupes l'avait amené également à contester d'autres utopies, politiques 
celles-ci. Ainsi, expliquait-il, si les moyens utilisés par les bolcheviques étaient les seuls réalistes pour se débarrasser des seigneurs capitalistes, rien ne garantissait que les nouveaux oligarques communistes n'allaient pas, à leur tour, exercer leur pouvoir égoïste (Niebuhr 1932, 21). Au-delà même de ses doutes sur la capacité du groupe à s'élever au-dessus de ses intérêts, il fustigeait aussi la vision communiste selon laquelle la destruction des structures de propriété capitaliste créerait un homme nouveau capable de vivre en accord parfait avec la volonté collective de la société, y voyant une « interprétation romantique des possibilités de la nature humaine elle-même »(Niebuhr 1932, 194). Et il laissait déjà entendre dans cet ouvrage que la vision d'une société juste et pacifique, "comme toutes les visions vraiment religieuses pouvait être en partie mais jamais entièrement réalisée dans l'histoire » (Niebuhr 1932, 22).

Pourtant il se refusait encore à tirer les conclusions qu'impliquait une telle vision relativiste et pessimiste des potentialités de la société humaine. Il ne le faisait pas car il estimait que la dimension utopique-qu'il qualifiait de religieuse, ou d'eschatologique - du marxisme était la force motrice du projet socialiste. En effet :

Once the religious quality of the proletarian creed is abandoned and the eschatological emphasis in Marxism is disavowed, evolutionary socialism may easily lose the furious energy which alone is capable of moving against the stubborn inertia of society (Niebuhr 1932, 222).

Et c'est sa crainte de l'inertie sociale qui lui faisait alors conclure :

In the task of redemption the most effective agents will Be men who have substituted some new illusions for the abandoned ones. The most important of these illusions is that the collective life of mankind can achieve perfect justice. It is a very valuable illusion for the moment; for justice cannot be approximated if the hope of its perfect realization does not generate a sublime madness in the soul. Nothing but madness will do battle with malignant power (...). The illusion is dangerous because it encourages terrible fanaticisms. It must therefore be brought under the control of reason. One can only hope that reason will not destroy it before its work is done (Niebuhr 1932, 277).

C'est sur cette tension que se refermait son ouvrage: la vision socialiste était une illusion, mais cette illusion était nécessaire afin de pousser les hommes à agir pour n'en atteindre au mieux qu'une approximation. Si pendant un temps il feignit de ne pas voir la contradiction qu'il y avait à fonder une politique réaliste sur une illusion, il abandonna bientôt la position de Moral Man face à l'évolution des évènements internationaux.

Son tournant théologique orthodoxe fut en effet motivé en grande partie par l'évolution de la situation européenne après la victoire d'Hitler qui lui faisait craindre pour la survie du christianisme lui-même. Il tira un bilan de faillite des socialistes allemands qui avaient facilité la montée d'Hitler par leur attitude légaliste. Mais s'il jugeait que les communistes avaient, eux, fait les bons choix quant aux moyens de lutte, il ne pouvait se résoudre à les considérer comme une alternative crédible car ils réduisaient la vie à la politique, la privant de sa dimension spirituelle essentielle. Face à cette impasse le christianisme prophétique lui apparut de plus en plus comme la seule planche de salut (Fox 1985, 148-149).

Il suivait là une évolution déjà à l'œuvre dans certains cercles protestants, sous l'influence du théologien suisse Karl Barth en Europe et de son propre frère, H. Richard Niebuhr, et certains de ses amis au séminaire de Yale. Karl Barth, engagé dans le socialisme continental à la veille de la guerre, s'était détourné de la politique après le 
vote des crédits de guerre par les socialistes allemands en 1914, revenant à une lecture orthodoxe des Ecritures, réintroduisant fermement la notion de transcendance et le pouvoir absolu de la parole de Dieu qui était au-delà de toutes les possibilités humaines et ne pouvait donc se réaliser dans l'histoire. Toute tentative de le faire ne pouvait que corrompre la parole divine et Barth appelait donc les chrétiens à se détourner des contingences temporelles. La "grâce de ne rien faire", c'est aussi la conclusion à laquelle était arrivé Richard Niebuhr qui, comme son frère, avait milité au sein du parti socialiste jusqu'en 1935, et appliqué aussi les outils de la sociologie marxiste dans son étude classique des institutions religieuses américaines, The Social Sources of Denominationalism (1929). Plus porté sur la théologie pure que son frère, depuis le début des années 1930 il avait engagé, sous l 'influence de l'orthodoxie de Barth, une critique radicale de toute l'évolution des Eglises aux Etats-Unis, revenant aux sources de la Réforme et réhabilitant Jonathan Edwards, rejetant ainsi le long cheminement opéré par le protestantisme évangélique américain pour accommoder la doctrine à l'évolution historique (Richard Niebuhr 1937 1988). Dans un ouvrage publié avec deux de ses collègues de Yale, William Pauck et Francis P. Miller, il établissait une claire distinction entre l'Eglise et le monde et affirmait que :

If the Church has no other plan to offer to men than one of deliverance by love, education, idealism or planned economy, it really has no existence as a Church and should resolve itself into a political party or a school (Niebuhr, Pauck, Miller 1935).

Or, pour le chrétien la loyauté envers Dieu était la condition même de l'existence, et l'Eglise, communion de confessants, devait donc clairement se dresser contre le monde, comme le proclamait d'ailleurs le titre de l'ouvrage.

On sent l'influence de l'orthodoxie barthienne dans les écrits théologiques de Reinhold Niebuhr à partir de 1935. Si le Social Gospel avait mis l'accent sur la relation de l'homme avec les autres hommes, Reinhold Niebuhr s'efforçait désormais de rétablir la relation plus fondamentale entre l'homme et Dieu. Or cette relation était marquée par le péché originel qui signalait la révolte de l'homme contre Dieu, sa volonté de donner un sens absolu à sa finitude et, en quelque sorte, de se substituer à Dieu à la fois comme créateur et objet de sa propre adoration (Niebuhr 1935). La réintroduction de la nature transcendantale du Royaume et du poids du péché dans le destin humain lui permettait de donner un soubassement théologique orthodoxe aux doutes qu'il entretenait depuis un certain temps sur la capacité de réaliser l'idéal de justice dans la société. Le problème ne venait plus en effet avant tout de l'égoïsme des groupes sociaux, mais de la nature peccamineuse (sinful) de l'homme, thème qui devint dès lors le leitmotiv de ses écrits. Et il en concluait que si l'individu pouvait se sauver en acceptant la grâce de l'amour divin, cet amour ne pouvait trouver son expression dans les relations entre les hommes, "marquées de façon indélébile par la propension humaine au péché et à l'erreur » (Niebuhr 1941, 148).

La question qui nous intéresse ici est de comprendre les implications politiques de ce tournant orthodoxe dans les années du second New Deal. Contrairement à Barth et à son frère, en effet, Reinhold Niebuhr ne pouvait se résoudre à l'inaction. Son nouveau réalisme théologique vint en fait renforcer, en le modifiant substantiellement, son réalisme politique. Le christianisme prophétique auquel il adhérait désormais ne se désintéressait pas des questions sociales mais, en rappelant sans cesse les limites absolues de l'expérience humaine, il ne devait plus dessiner un idéal susceptible de lancer les hommes dans l'action, mais déterminer face aux options politiques en 
présence laquelle était la moins mauvaise. L'absolutisme théologique nourrissait ainsi un relativisme politique qui explique son ralliement au New Deal.

Si son tournant théologique orthodoxe avait été motivé avant tout par les évènements internationaux, l'évolution de la situation aux Etats-Unis vint le conforter. Comme il l'avait craint dans Moral Man, il était difficile de divorcer l'action politique concrète de l'idéal qui la motivait et sa mise en garde contre «les illusions nécessaires » n'avait rencontré qu'hostilité dans les milieux de gauche, laïcs ou religieux. Le climat était plutôt à la radicalisation comme le signalait la scission de gauche du parti socialiste en 1934, l'influence locale croissante du parti communiste et l'engagement d'un certain nombre de pasteurs politisés dans les luttes syndicales qui ne se donnaient pas comme objectif une simple approximation de justice sociale. C'est pour contrer cette tendance qu'il créa dès l'automne 1934 la Fellowship of Socialist Christians avec certains de ses amis de Union Theological Seminary et publia, à partir de la fin 1935, la revue Radical Religion. Les noms choisis pour l'organisation et sa publication n'étaient pas dus au hasard. Comme le notait l'éditorial du premier numéro de Radical Religion, si l'organisation réaffirmait nettement son engagement anticapitaliste, elle soulignait que sa "loyauté première allait au christianisme " et regrettait que "de trop nombreux jeunes pasteurs aient été entraînés vers une capitulation totale face aux dogmes marxistes » (Radical Religion 1935). L'objectif de l'organisation, au sein de laquelle Reinhold Niebuhr concentra dès lors le gros de son activité, était, sans détourner ces pasteurs de l'action, de leur en faire admettre ses limites insurmontables, ce qui n'était évidemment pas une tâche aisée, et son influence ne fut jamais très grande. L'action de Niebuhr était entravée par une autre tendance, dans le sens opposé celle-là, qui voyait un nombre croissant de militants réformistes et radicaux se rapprocher du New Deal. Norman Thomas lui-même, après son échec électoral de 1936 et face à la popularité croissante de Roosevelt au sein de la classe ouvrière se mit à parler plus souvent de "démocratie industrielle» que de socialisme (Gutman 1992, vol. 2 : 402-403). Il était évident que grâce au New Deal, le gouvernement jouait un rôle de plus en plus important dans la formation de la «foi commune» des Américains, pour reprendre l'expression de John Dewey, une foi faite d'idéaux démocratiques et égalitaires dont il promettait la réalisation dans l'histoire (Dewey 1934). Pour Reinhold Niebuhr, la voie était donc étroite entre sa fonction prophétique de chrétien qui devait mettre en garde contre toutes les illusions de l'action humaine et son réalisme politique qui le forçait à admettre que, contrairement à ses craintes du début de la décennie, le New Deal n'avait pas mené à la catastrophe et, au contraire semblait devoir être en mesure d'épargner aux Etats-Unis les tragédies qui s'annonçaient en Europe. Si le réalisme chrétien consistait désormais à choisir le moindre mal, dans le contexte de la seconde moitié des années trente, le choix devait à l'évidence se porter sur le président démocrate et son programme.

Et, en effet, dès son second numéro, Radical Religion modérait sa position anticapitaliste, annonçant que

Historic situation in Western nations does not offer the possibility of breaking through to a new society. It offers only the immediate possibility of defending democratic institutions, however corrupted, against the peril of fascism (Radical Religion 1935/36).

Pourtant, en 1936, Niebuhr appela à nouveau à voter pour le candidat socialiste et pour quelque temps encore continua à défendre l'idée d'un troisième parti (Niebuhr 1937a). Étant donné ses virulentes critiques de la période précédente, l'ajustement nécessitait à 
l'évidence du temps et quelques contorsions. Mais on note graduellement que Radical Religion parle de plus en plus de réforme du système, de planification sans expropriation, du poids que la classe ouvrière pourrait faire peser à l'intérieur des institutions. C'est l'offensive conservatrice du monde des affaires contre Roosevelt à partir de 1938 qui lui offrit finalement l'occasion de le défendre comme le moindre mal. Au printemps 1938, Radical Religion comparait encore le New Deal à une dose d'insuline pour un système anémié, mais notait que FDR valait quand même mieux que ses ennemis. L'année suivante la position était plus tranchée. La revue appelait à défendre les acquis du New Deal contre les conservateurs et parlait du Wagner Act comme de la "Tchécoslovaquie de notre politique nationale ». Il fallut le début de la guerre en Europe, pour que Niebuhr se résolve à soutenir le président démocrate, tout en mettant en garde contre le danger de se laisser « duper » par lui (Niebuhr 1939b).

Mais Niebuhr abandonna vite ses réserves. Au cours de la campagne électorale de 1940, dominée par les questions de politique étrangère, le choix s'imposait sans hésitation: avec sa politique de préparation militaire Roosevelt lui paraissait autrement réaliste que l'isolationniste Norman Thomas et le parti socialiste, dont il démissionna en mai 1940. Niebuhr dénonçait désormais les actions syndicales dans les industries militaires et défendait le bilan de Roosevelt qu'il n'avait pourtant cessé de combattre pendant huit ans, affirmant que son administration était en fait plus démocratique que les forces sociales démocratiques représentées par la classe ouvrière, ce qui, à son avis, démentait clairement les "théories faciles" (qu'il avait longtemps défendues) selon lesquelles « le gouvernement était un comité de la classe dominante servant à écraser les dominés " (Niebuhr 1940b). L'urgence était désormais à l'effort de guerre et non plus au jugement prophétique et une certaine dose d'illusion était peut-être redevenue nécessaire.

41 Certains historiens de la religion ont noté que les années trente représentent l'aboutissement d'un processus, en cours depuis le début du siècle, de séparation inexorable entre le protestantisme et le pouvoir (Hammond 1992, 28). John Higham a confirmé cette analyse a un autre niveau, en montrant, lors d'un colloque à l'Université Paris VII en 2000, que nombre des programmes du New Deal avaient été largement influencés par les approches plus collectivistes des questions sociales apportées par les immigrants européens de culture catholique ou juive (Higham 2001). Le Social Gospel avait tenté de réagir à cette marginalisation politique du protestantisme, en niant toute fonction distincte au politique et proposant la christianisation de la société comme solution à ses maux. C'est en critiquant son idéalisme que Reinhold Niebuhr développa sa démarche réaliste qui, d'un côté, reconnaissait l'autonomie du politique dans la gestion des rapports entre les hommes mais qui, finalement, le dévalorisait en insistant sur ses limites intrinsèques. Quant aux masses protestantes durant les années trente, il semble qu'elles n'aient suivi ni les idéalistes du Social Gospel, ni les prophètes du réalisme chrétien, pratiquant une religion personnelle et réagissant au New Deal en fonction de leurs intérêts de classe les plus immédiats. Dans ce sens, si la réflexion et l'action de Reinhold Niebuhr durant les années 1930 marquent une étape importante dans la tentative de redéfinition des rapports entre religion et politique, il n'a pas apporté de réponse définitive aux questions qu'il posait, comme on peut le constater jusqu'à ce jour. 


\section{BIBLIOGRAPHIE}

ABRAMS, Ray. Preachers Present Arms !, Scottdale, PA : Herald Press, 1933.

BALTZELL, E. Digby. The Protestant Establishment. New York : Random House, 1964.

BENNETT, John C. « A Changed Liberal, but still a Liberal. » Christian Century, 8 février 1939.

CARTER, Paul. The Decline and Revival of the Social Gospel, 1920-1940. Ithaca, N.Y. : Cornell University Press, 1956.

CARWARDINE, Richard J. Evangelicals and Politics in Antebellum America. New Haven : Yale University Press, 1993.

Christian Century. 22 mars 1931

Christian Century. « Methodist Money Speaks. » 14 août 1935.

DEWEY, John. A Common Faith. New Haven \& London : Yale University Press, 1934.

DOUGLASS, H. Paul, BRUNNER, Edmund deS. The Protestant Churches as a Social Institution. New York : Russell \& Russell, 1935.

FEDERAL COUNCIL OF CHURCHES. The Church and Social Reconstruction. New York : FCC, 1919. , « The Churches and the New Deal. » Federal Council Bulletin, septembre 1933.

Council Bulletin, octobre 1933.

FOX, Richard Wigham. Reinhold Niebuhr. A Biography, New York : Pantheon Books, 1985.

GUSTAFSON, Merlin. « Franklin D. Roosevelt and his Protestant Constituency », Journal of Church and State, été 1981, 285-297.

GUTMAN, Herbert G. ed. Who Built America? Working People and the National Economy, Politics, Culture, and Society. 2 vols., New York, Pantheon Books, 1992.

HAMMOND, Phillip E. The Protestant Presence in the Twentieth Century. Albany, NY : State University of New York Press, 1992.

HIGHAM,John. " The Long Road to the New Deal » in John HIGHAM, Hanging Together, Unity and Diversity in American Culture, ed. By Carl Guarneri, New Haven, Yale University press, 2001, pp. 199-221. Cet article a été repris in Peuples, Nation, Lien Social. Ed. Nathalie Caron \& Naomi Wulf, Paris :Syllepses, 2004.

McLOUGHLIN, William G. Revivals, Awakenings and Reform. Chicago : University of Chicago Press, 1978.

MENENDEZ, Albert J. Religion at the Polls. Philadelphie : Westminster Press, 1972.

MEYER, Donald. The Protestant Search for Political Realism, 1919-1941. Middletown, Conn. : Wesleyan University Press, $2^{\text {nd }}$ ed., 1988.

MILLER, Robert M. American Protestantism and Social Issues, 1919-1939. Chapel Hill : University of North Carolina Press, 1958.

MORRISON, Charles Clayton. « A Political Party Without Candidates. » Christian Century, 25 mai 1932a. 
«When Will America Begin to Plan? » Christian Century, 9 novembre $1932 b$.

MUELDER, Walter G. Methodism and Society in the Twentieth Century. New York \& Nashville : Abingdon Press, 1961.

NIEBUHR, Reinhold. Does Civilization Need Religion? New York : Scribner's, 1927a.

--------. Moral Man and Immoral Society. Louisville, Londres : Westminster John Knox Press, 1932, 2001.

--------. Reflections on the End of an Era. New York : Scribner's, 1934c.

---------. An Interpretation of Christian Ethics. New York : Harper \& Brothers, 1935.

Christianity and Power Politics. New York : Scribner's, 1940.

. « The Church and the Middle Class. » Christian Century, 7 décembre 1922.

. « What the War did to my Mind. » Christian Century, 27 septembre 1928a.

---------. « The Confession of a Tired Radical. » Christian Century, 30 août $1928 b$.

---------.. « Anglo-Saxon Protestant Domination. » World Tomorrow, novembre 1928c.

. «Protestantism and Prohibition. » The New Republic, 24 octobre 1928d.

« Why I leave the F.O.R.. » Christian Century, 3 janvier 1934a.

. « The Fellowship of Socialist Christians. » World Tomorrow, 14 juin $1934 \mathrm{~b}$.

. « Roosevelt and the Sharecroppers. » Radical Religion, printemps 1937a.

---------.. « A New Party ? » Radical Religion, automne 1937b.

--------. « The Socialist Party and the Labour Movement. » Radical Religion, hiver 1937c.

----------. « Peace and the Liberal Illusion. » The Nation, 28 janvier 1939.

---------. « Ten Years that Shook my World. » Christian Century, 26 avril 1939a.

---------. « We must not be Beguiled by Mr. Roosevelt. » Radical Religion, automne $1939 \mathrm{~b}$.

---------. « Marxists are Taking Stock. » Christianity and Society, printemps 1940a.

--------. « Wilkie and Roosevelt. » Christianity and Society, automne $1940 \mathrm{~b}$.

NIEBUHR, H. Richard. The Kingdom of God in America. Middletown, Conn. : Wesleyan University Press, (1937) 1988.

-. Social Sources of Denominationalism. New York : H. Holt \& Co., 1929.

. MILLER, Francis, PAUCK, Wilhelm. The Church Against the World. Chicago : Willett, Clark $\& C^{\circ}, 1935$.

PAGE, Kirby. Imperialism and Nationalism. New York : Scribner's, 1925.

PELLS, Richard H. Radical Visions and American Dreams. New York : Harper \& Row, 1974.

POPE, Liston, Millhands and Preachers, New Haven, Yale University Press, 1942.

Radical Religion. 31 août 1933

Radical Religion. Hiver 1935/36.

World Tomorrow. 31 août 1933. 


\section{RÉSUMÉS}

Le New Deal est le premier mouvement réformiste d'ampleur dans l'histoire des Etats-Unis qui n'ait pas été inspiré, ni soutenu, par les forces du protestantisme. Si une majorité des protestants et de leur clergé se sont opposés au New Deal à partir de positions conservatrices, le théologien Reinhold Niebuhr en a développé au départ une virulente critique de gauche avant de finalement rallier le président démocrate à la fin des années trente. Nourrie par le rejet de l'idéalisme du Social Gospel, incapable à ses yeux de comprendre les rapports sociaux en terme de classe et de pouvoir, la critique de Niebuhr emprunte les outils d'analyse marxistes pour développer une approche réaliste, tant du point de vue des fins que des moyens de la lutte politique. Le New Deal lui apparaît avant tout comme un programme visant à sauver le capitalisme, alors que la société fondée sur la justice qu'il envisage passe par la socialisation des moyens de production qui ne pourra émerger qu'à travers des conflits sociaux au cours desquels les moyens de lutte même violents sont justifiés. Mais sous l'influence des évènements en Europe au lendemain de la victoire d'Hitler, Reinhold Niebuhr se tourne au milieu des années trente vers une réflexion théologique inspirée de l'orthodoxie de Karl Barth et de son frère Richard Niebuhr, qui l'amène à réintroduire le péché comme la limite absolue de toute l'expérience humaine, interdisant d'envisager la création d'une société de paix et de justice. Ce nouvel absolutisme théologique nourrit un relativisme politique, le réalisme chrétien consistant désormais à choisir la moins mauvaise des options en présence. De ce point de vue, le président démocrate qui appelle à la préparation militaire s'impose désormais comme le choix le plus réaliste face à la montée du fascisme en Europe.

The New Deal was the first major reformist experiment in the history of the United States that was neither inspired nor supported by Protestantism. While a majority of Protestants and their clergy opposed Franklin Roosevelt from a conservative position, the theologian Reinhold Niebuhr developed a virulent left-wing criticism of his policies, before finally rallying to his support at the end of the decade. Rejecting the idealism of the Social Gospel, unable to understand social relationships in term of class interests and power, Niebuhr used Marx's analytical tools to develop a more realistic and efficient political approach. He saw the New Deal as a program aiming to save capitalism through state action, whereas the society based on social justice that he envisaged required the socialisation of the means of production which could be brought about only through social conflicts that may require the use of violence. However, under the influence of events in Europe after the rise of Hitler to power, he turned increasingly to theology. Inspired by the neo-orthodoxy of Karl Barth and of his own brother, Richard Niebuhr, he reintroduced sin as the absolute limit of human action. Because of man's sinful nature it was impossible to create a society based on justice and peace. His new theological absolutism led to political relativism. Indeed, realism now meant that in politics a Christian could only choose between the lesser of two evils. From this point of view the Democratic president and his preparedness campaign appeared as the only realistic choice in the context of the rise of fascism in Europe.

\section{INDEX}

Keywords : Reinhold Niebuhr, Social Gospel, Liberal Protestantism, Radicalism, Christian realism Mots-clés : Evangile social, Protestantisme libéral, Radicalisme, Réalisme chrétien 
AUTEUR

ISABELLE RICHET

Université Paris VII 